\title{
Optimal replacement policy of products with repair-cost threshold after the extended warranty
}

\author{
Lijun Shang and Zhiqiang Cai* \\ School of Mechanical Engineering, Northwestern Polytechnical University, Xi'an 710072, China
}

\begin{abstract}
The reliability of the product sold under a warranty is usually maintained by the manufacturer during the warranty period. After the expiry of the warranty, however, the consumer confronts a problem about how to maintain the reliability of the product. This paper proposes, from the consumer's perspective, a replacement policy after the extended warranty, under the assumption that the product is sold under the renewable free replacement warranty (RFRW) policy in which the replacement is dependent on the repair-cost threshold. The proposed replacement policy is the replacement after the extended warranty is performed by the consumer based on the repair-cost threshold or preventive replacement $(\mathrm{PR})$ age, which are decision variables. The expected cost rate model is derived from the consumer's perspective. The existence and uniqueness of the optimal solution that minimizes the expected cost rate per unit time are offered. Finally, a numerical example is presented to exemplify the proposed model.
\end{abstract}

Keywords: extended warranty, cost threshold, minimal repair, cost-based replacement, age-based replacement.

DOI: $10.21629 / J S E E .2017 .04 .12$

\section{Introduction}

Warranty is a marketing tool, and can benefit simultaneously the manufacturer and the customer. From the manufacturer's perspective, the warranty can make the manufacturer gain more profit and a favourable reputation. From the customer's perspective, the warranty makes product quality more reliable. The warranty has recently been a hot research topic due to its importance. A great many of warranty policies were widely researched by academic researchers or practitioners. The earlier and completer warranty policies were found in [1], and some recent research can be found in [2-6].

With the development of technology, some products are

\footnotetext{
Manuscript received April 07, 2016.

*Corresponding author.

This work was supported by the National Natural Science Foundation of China (71471147; 71631001), the Basic Research Project of Natural Science in Shaanxi Province (2015JQ7273) and the 111 Project (B13044).
}

increasingly complex and multi-functional. Thus, the repair cost resulted from failure is dependent on the damage extent of failure. In other words, when a failure occurs, a harsher damage leads to a greater repair cost. If this repair cost approaches or exceeds a cost limit (e.g., sale price or production cost), the manufacturer or the consumer would like to replace the failed product by a new identical one rather than repair it. This implies that if the repair cost approaches or exceeds the certain threshold, the failed product is replaced; otherwise, the failed product is repaired.

This type of the cost limit was early used to investigate some warranty problems in $[2,7,8]$. However, these literature concentrated only on the non-renewable warranty policy, did not consider the extended warranty and considered the repair-cost limit as a unique decision variable. In practice, the renewable free replacement warranty (RFRW) policy is one of the most popular warranty policies, because most customers prefer to obtain a new replacement at no cost when the product fails during the warranty period. Considering this preference, the manufacturer is very willing to provide the RFRW policy for the customer in order to increase sales volumes of the product. But this action produces higher replacement frequency since any failure during the warranty period is required to be removed by using an identical one to replace. This makes the manufacturer incur more replacement cost. In order to overcome this shortcoming, we consider an RFRW policy with the repair-cost threshold in this paper, where the failed product is replaced if the repair cost resulted from failure exceeds the repair-cost threshold and otherwise this failure is removed by the minimal repair. Obviously, this warranty policy not only keeps the replacement action and but also reduces the replacement cost.

For the product sold under a warranty, besides, although reliability is maintained by the manufacturer during the warranty, the consumer still confronts a problem about how to maintain reliability during the post-warranty period. Because of the increasing expected cost rate during 
the post-warranty period, recently, maintenance policies after the warranty expiration have attracted increasing attention. This type of research was found early in [9], and the subsequent research can be found in [6-16]. These literatures focused mainly on three types of maintenance policies after the warranty expiration. The first type is agebased maintenance or/and preventive replacement (PR), see for example [6,10-16]. The second type is cost-based corrective replacement (CR), which can be found in $[7,8]$. The third type is the combination of the extended warranty and age-based replacement, which can be found in [6]. It is worth pointing out that there is few literature on the postwarranty maintenance policy, where a combination of the age-based PR and the cost-based CR is considered. Compared with the traditional age replacement policy, the advantage of this combination policy is to reduce replacement frequency.

The main objective of this paper is, from the consumer's point of view, to propose an optimal replacement policy after the expiry of the extended warranty, under the assumption that the product is sold under the RFRW policy with the repair-cost threshold. However, the approach of this study is different from that of the existing works. Firstly, the warranty policy considered in this paper is the RFRW policy with the repair-cost threshold. This differs from $[10,11,14-16]$, where each failure during the warranty requires replacement. That is, each failure during the warranty period is removed by using a new identical one to replace. During the PR period, secondly, the consumer performs at his/her expense replacement for the failed product based on the repair-cost threshold or the PR age. This is different from [7,8], where the CR related to repair-cost threshold was only considered and the PR related to age was not considered.

The remainder of this paper is organized as follows. Section 2 gives the problem descriptions and assumptions. Section 3 presents the expected cost rate model. In Section 4, a numerical example is offered. Finally, we conclude this study with a brief discussion in Section 5 .

\section{Problem descriptions}

\subsection{Problems}

We first assume the product is sold under the proposed warranty policy, i.e., the RFRW policy with the repair-cost threshold, then deal with a problem about the consumer how to maintain the reliability of the product when the warranty expires. The detailed information is as follows. The reliability of the product is maintained by the manufacturer during the basic warranty period $w$. When the basic warranty expires, the consumer purchases $k$ units of the extended warranty, where the failed product is restored to the condition "as bad as old" regardless of the repair cost. Subsequently, the product with the past age $w+k \xi$ operates during the PR period $T$, where the consumer estimates the repair cost resulted from each failure. If the repair cost $Y_{l}$ resulted from the $l$ th failure exceeds the repair-cost threshold $\Re$ predetermined by the consumer, then the consumer will carry out the $\mathrm{CR}$ in which the failed product is replaced by a new identical one. Otherwise, the consumers will perform the PR at the age $T$. Obviously, each purchase of the consumer means one renewal.

Similar to $[6,10,14]$, we define the life cycle as an interval from the installment instant of a new product purchased by the consumer to the replacement instant of this product at the consumer's expense, which is diagrammed in details in Fig. 1.

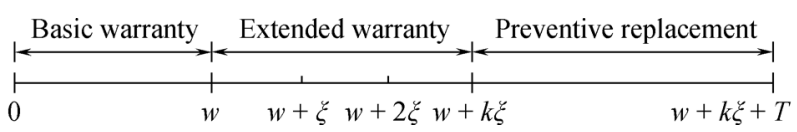

Fig. 1 Life cycle of the product

Therefore, our problem is, from the consumer's perspective, to determine the repair-cost threshold $\Re$ and the PR age $T$, which will balance the CR cost and the PR cost. For this, we first compute the expected length $\mathrm{E}[L]$ of the life cycle and the expected total cost $\mathrm{E}[C(L)]$ during the life cycle, then compute the expected cost rate based on the renewal rewarded theorem in [17], as

$$
\lim _{t \rightarrow \infty} \frac{D(t)}{t}=\frac{\text { Expected cost in one cycle }}{\text { Mean time of one cycle }} .
$$

\subsection{Assumptions}

(i) All claims are valid and accepted during the basic warranty and the extended warranty period.

(ii) The repair time and replacement time are neglectful.

(iii) The manufacturer implements minimal repair for the failed product at no charge to the consumer during the extended warranty period $k \xi$.

(iv) The distribution function of lifetime does not depend on the distribution function of the repair cost.

(v) The distribution function of the lifetime and the distribution function of the repair cost are continuous and are known to the consumer.

(vi) The repair-cost threshold $\theta$ predetermined by the manufacturer is also known to the consumer.

\section{Formulation of cost rate model}

\subsection{Preliminaries}

$\theta$ : repair-cost threshold predetermined by the manufacturer; 
$\Re$ : repair-cost threshold predetermined by the consumer.

Let the random variable $X$ be the time to failure, then its characteristics are described as follows.

$F(x)$ : distribution function;

$\bar{F}(x)$ : reliability function, where $\bar{F}(x)=1-F(x)$;

$\gamma(x)$ : failure rate function, where $\gamma(x)=\frac{\mathrm{d} F(x)}{\bar{F}(x) \mathrm{d} x}$.

Let the random variable $Y$ be the repair cost, then its characteristics are described as follows.

$G(y)$ : distribution function;

$\bar{G}(y)$ : reliability function, where $\bar{G}(y)=1-G(y)$.

Let the random variable $X^{*}$ be the interval between two consecutive replacements during the basic warranty period $w$, then its characteristics are described as follows.

$\bar{F}_{\theta}(x)$ : reliability function, where $\bar{F}_{\theta}(x)=$ $\exp \left(-\bar{G}(\theta) \int_{0}^{x} \gamma(u) \mathrm{d} u\right)=[\bar{F}(x)]^{\bar{G}(\theta)} ;$

$F_{\theta}(x)$ : distribution function, where $F_{\theta}(x)=1-\bar{F}_{\theta}(x)$.

\subsection{The expected length of the life cycle}

During the basic warranty period $w$, if the repair cost $Y_{i}$ resulted from the $i$ th failure exceeds the repair-cost threshold $\theta$, then the manufacturer will replace this failed product. So we denote the lifetime $X^{0}$ of the replaced product as $X_{i} \mid X_{i} \leqslant w, Y_{i} \geqslant \theta(i=1,2,3, \cdots)$, then the distribution function of $X^{0}$ is obtained as

$$
F_{b w}(x)=\left\{\begin{array}{l}
1, \quad x>w \\
\frac{F_{\theta}(x)}{F_{\theta}(w)}, \quad 0 \leqslant x \leqslant w \\
0, \quad \text { otherwise }
\end{array}\right.
$$

where $X_{i}$ is the occurrence time of the $i$ th failure during the basic warranty period $w$.

Let the random variable $\eta_{1}-1$ be the number of replacements until the first product surviving the basic warranty period $w$ is obtained, then $\eta_{1}$ follows the geometric distribution as

$$
P_{1}(n)=P\left\{\eta_{1}=n\right\}=\left[F_{\theta}(w)\right]^{n-1} \cdot\left[\bar{F}_{\theta}(w)\right]
$$

Furthermore, the expected number of replacements during the basic warranty period $w$ is given by

$$
\mathrm{E}\left[\eta_{1}-1\right]=\frac{1-[\bar{F}(w)]^{\bar{G}(\theta)}}{[\bar{F}(w)]^{\bar{G}(\theta)}}=\frac{F_{\theta}(w)}{\bar{F}_{\theta}(w)}
$$

Obviously, when $\theta=0$, then $\mathrm{E}\left[\eta_{1}-1\right]=\frac{F(w)}{\bar{F}(w)}$, which is the expected number of replacements under the traditional RFRW policy that is found in $[6,14]$. So we have the following proposition.
Proposition 1 For a given warranty period $w$, the expected number $\frac{F_{\theta}(w)}{\bar{F}_{\theta}(w)}$ of replacements under the warranty policy proposed in this paper is smaller than the expected number $\frac{F(w)}{\bar{F}(w)}$ of replacements under the traditional RFRW policy, i.e., $\frac{F_{\theta}(w)}{\bar{F}_{\theta}(w)}<\frac{F(w)}{\bar{F}(w)}$.

Proof Let $h(x)=\frac{x}{1-x}$, then obviously $h(x)$ increases with respect to $x$. In addition, $[\bar{F}(w)]^{x}(0<$ $\bar{F}(w)<1)$ decreases with respect to $x$, so $1-$ $[\bar{F}(w)]^{\bar{G}(\theta)}<1-\bar{F}(w)$ when $0<\bar{G}(\theta)<1$. Thus, $\frac{1-[\bar{F}(w)]^{\bar{G}(\theta)}}{1-\left(1-[\bar{F}(w)]^{\bar{G}(\theta)}\right)}<\frac{1-\bar{F}(w)}{1-(1-\bar{F}(w))}$, i.e., $\frac{F_{\theta}(w)}{\bar{F}_{\theta}(w)}<$ $\frac{F(w)}{\bar{F}(w)}$

Let $\left\{X_{j}\right\}\left(j=1,2, \cdots, \eta_{1}-1\right)$ be an independent and identically distributed (i.i.d.) sequence of random variables subjected to $F_{b w}(x)$, then the expected life length of the product replaced during the basic warranty period $w$ is calculated as

$$
\mathrm{E}\left[X_{j}\right]=\frac{\int_{0}^{w} x \mathrm{~d} F_{\theta}(x)}{F_{\theta}(w)} .
$$

The length of the actual warranty servicing period is expressed as

$$
W=\sum_{j=1}^{\eta_{1}-1} X_{j}+w
$$

As $\eta_{1}$ is also a stopping time, by Wald's equation in [18], (3) and (4), the expected length of the actual warranty servicing period is obtained as

$$
\mathrm{E}[W]=\mathrm{E}\left[\eta_{1}-1\right] \cdot \mathrm{E}\left[X_{j}\right]+w=\frac{\int_{0}^{w} \bar{F}_{\theta}(x) \mathrm{d} x}{\bar{F}_{\theta}(w)} .
$$

After the expiry of the extended warranty, the product with the past age $w+k \xi$ operates during the PR pe$\operatorname{riod} T$, where the consumer will replace the failed product by a new identical one if the repair cost $Y_{l}$ resulted from the $l$ th failure exceeds the repair-cost threshold $\Re$. Hence, we denote the lifetime $X^{1}$ of the product replaced as $X_{l} \mid X_{l}>w+k \xi, Y_{l} \geqslant \Re(l=1,2,3, \cdots)$, then $X^{1}$ follows the reliability function, as

$$
\bar{F}_{\text {aew }}(x)=\frac{[\bar{F}(w+k \xi+x)]^{\bar{G}(\Re)}}{[\bar{F}(w+k \xi)]^{\bar{G}(\Re)}}
$$

where $X_{l}$ is the occurrence time of the $l$ th failure during the PR period $T$. 
During the PR period $T$, additionally, the consumer will implement $\mathrm{CR}$ for the failed product if the repair cost $Y_{l}$ resulted from the $l$ th failure exceeds the repair-cost threshold $\Re$; otherwise, the consumer will perform PR for the product at the PR age $T$. Let the random variable $Z^{*}$ be the occurrence time of CR. During the PR period $T$, therefore, the occurrence time of replacement is $Z_{r}=\min \left(Z^{*}, T\right)$, then its expectation can be given by

$$
\mathrm{E}\left[Z_{r}\right]=\int_{0}^{T} \bar{F}_{\text {aew }}(x) \mathrm{d} x
$$

By Subsection 2.1, the length of the life cycle is written as

$$
L=W+k \xi+Z_{r} .
$$

By (6) and (8), finally, the expected length of the life cycle can be represented as

$$
\begin{gathered}
\mathrm{E}[L]=\mathrm{E}[W]+k \xi+\mathrm{E}\left[Z_{r}\right]= \\
\frac{\int_{0}^{w} \bar{F}_{\theta}(x) \mathrm{d} x}{\bar{F}_{\theta}(w)}+k \xi+\int_{0}^{T} \bar{F}_{a e w}(x) \mathrm{d} x .
\end{gathered}
$$

\subsection{The expected cost during the life cycle}

During the basic warranty period $w$, let the random variable $\eta_{2}-1$ be the number of minimal repairs between two consecutive replacements, so the variable $\eta_{2}$ has the following the geometric distribution, as

$$
P_{2}(n)=P\left\{\eta_{2}=n\right\}=[G(\theta)]^{n-1}[\bar{G}(\theta)]
$$

where $\bar{G}(\theta)$ is the probability that replacement occurs, where $\bar{G}(\theta)=1-G(\theta)$.

During the basic warranty period $w$, therefore, the expected number of minimal repairs between two consecutive replacements is given by

$$
\mathrm{E}\left[\eta_{2}-1\right]=\frac{G(\theta)}{\bar{G}(\theta)}
$$

Let $\left\{\eta_{2}^{j}\right\}\left(j=1,2, \cdots, \eta_{1}-1\right)$ be an i.i.d. sequence of random variables conforming to $P_{2}(n)$. Therefore, the total cost during the basic warranty period $w$ is computed as

$$
v(w)=\sum_{j=1}^{\eta_{1}-1}\left[c_{0} \cdot \eta_{2}^{j}\right]+c_{0} G(\theta) \int_{0}^{w} \gamma(x) \mathrm{d} x
$$

where $c_{0}$ is the unit failure cost during the basic warranty period $w$.
By (3) and (12), the expected total cost during the basic warranty period $w$ is given by

$$
\begin{gathered}
\mathrm{E}[v(w)]=\mathrm{E}\left[\sum_{j=1}^{\eta_{1}-1}\left[c_{0} \cdot \eta_{2}^{j}\right]+c_{0} G(\theta) \int_{0}^{w} \gamma(x) \mathrm{d} x\right]= \\
\mathrm{E}\left[\eta_{1}-1\right] \cdot \mathrm{E}\left[c_{0} \cdot \eta_{2}^{j}\right]+c_{0} G(\theta) \int_{0}^{w} \gamma(x) \mathrm{d} x= \\
\frac{F_{\theta}(w) c_{0}}{\bar{F}_{\theta}(w) \bar{G}(\theta)}+c_{0} G(\theta) \int_{0}^{w} \gamma(x) \mathrm{d} x .
\end{gathered}
$$

After the basic warranty expiration, the consumer purchases $k$ units of the extended warranty period, where the manufacturer immediately performs minimal repair for the failed product regardless of repair cost. Therefore, the expected total cost during the extended warranty period $k \xi$ is equal to the sum of the total cost incurred for purchasing $k$ units of the extended warranty and the total failure cost during the extended warranty period $k \xi$. Then it is computed by

$$
\mathrm{E}\left[C_{e}^{w}\right]=k c_{e}+c_{1} \int_{w}^{k \xi} \gamma(t) \mathrm{d} t
$$

where $c_{e}$ is the unit cost incurred for purchasing one unit of the extended warranty, and $c_{1}$ is unit failure cost during the extended warranty period $k \xi$.

During the PR period $T$, if the repair cost $Y_{l}$ resulted from the $l$ th failure does not exceeds the repair-cost threshold $\Re$, then the consumer will perform the minimal repair for the failed product at his/her expense. Denote the repair cost $Y^{*}$ as $Y_{l} \mid Y_{l} \leqslant \Re(l=1,2,3, \cdots)$, then $Y^{*}$ follows the distribution function, as

$$
G_{r}(y)=\left\{\begin{array}{l}
1, \quad y>\Re \\
\frac{G(y)}{G(\Re)}, \quad 0<y \leqslant \Re . \\
0, \quad \text { otherwise }
\end{array}\right.
$$

Therefore, the expected repair cost resulted from each failure during the PR period $T$ is represented as

$$
C_{R}=\mathrm{E}\left[Y^{*}\right]=\int_{0}^{\Re} y \mathrm{~d} G_{r}(y)=\frac{\int_{0}^{\Re} y \mathrm{~d} G(y)}{G(\Re)} .
$$

Further, if the repair cost $Y_{l}$ resulted from the $l$ th failure exceeds the repair-cost threshold $\Re$ during the PR period $T$, then the consumer will implement the CR with a cost $c_{r}$; otherwise the consumer will carry out the PR with a cost $c_{p}\left(c_{r}>c_{p}\right)$ at the PR age $T$. Therefore, the expected total cost during the PR period $T$ is calculated as 


$$
\begin{gathered}
\mathrm{E}[C(\Re, T)]= \\
\bar{F}_{\text {aew }}(T)\left(\left(c_{2}+C_{R}\right) G(\Re) \int_{0}^{T} \gamma_{e w}(u) \mathrm{d} u+c_{p}\right)+ \\
\int_{0}^{T}\left(\left(c_{2}+C_{R}\right) G(\Re) \int_{0}^{x} \gamma_{e w}(u) \mathrm{d} u+c_{r}\right) \mathrm{d}\left(1-\bar{F}_{\text {aew }}(x)\right)= \\
\left(c_{2}+C_{R}\right)\left[\int_{0}^{T} \bar{F}_{\text {aew }}(x) \gamma_{e w}(x) \mathrm{d} x-F_{\text {aew }}(T)\right]+ \\
c_{p} \bar{F}_{\text {aew }}(T)+c_{r} F_{\text {aew }}(T)
\end{gathered}
$$

where $c_{2}$ is the unit failure cost during the PR period $T$, and $\gamma_{e w}(x)=\gamma(w+k \xi+x)$.

Moreover, the expected total cost during the life cycle is equal to the sum of the expected total cost $\mathrm{E}[v(w)]$ during the basic warranty period $w$, the expected total cost $\mathrm{E}\left[C_{e}^{w}\right]$ during the extended warranty period $k \xi$ and the expected total cost $\mathrm{E}[C(T, \Re)]$ during the PR period $T$. Then it can be mathematically expressed as

$$
\begin{gathered}
\mathrm{E}[C(L)]=\mathrm{E}[v(w)]+\mathrm{E}\left[C_{e}^{w}\right]+\mathrm{E}[C(T, \Re)]= \\
M+k c_{e}+c_{1} \int_{w}^{k \xi} \gamma(t) \mathrm{d} t+ \\
\left(c_{m m}+C_{R}\right)\left[\int_{0}^{T} \bar{F}_{\text {aew }}(x) \gamma_{\text {ew }}(x) \mathrm{d} x-F_{\text {aew }}(T)\right]+ \\
c_{p} \bar{F}_{\text {aew }}(T)+c_{r} F_{\text {aew }}(T)
\end{gathered}
$$

where

$$
M=\mathrm{E}[v(w)]=\frac{F_{\theta}(w) c_{0}}{\bar{F}_{\theta}(w) \bar{G}(\theta)}+c_{0} G(\theta) \int_{0}^{w} \gamma(x) \mathrm{d} x .
$$

\subsection{The expected cost rate model}

The expected length of the life cycle and the expected total cost during the life cycle are derived respectively in (10) and (19). Based on the renewal rewarded theorem in [17], therefore, the expected cost rate is obtained as

$$
\begin{gathered}
D(\Re, T)=\frac{\mathrm{E}[C(L)]}{\mathrm{E}[L]}= \\
\frac{1}{\left(\int_{0}^{w} \bar{F}_{\theta}(x) \mathrm{d} x\right) /\left(\bar{F}_{\theta}(w)\right)+k \xi+\int_{0}^{T} \bar{F}_{\text {aew }}(x) \mathrm{d} x} \\
{\left[M+k c_{e}+c_{1} \int_{w}^{k \xi} \gamma(t) \mathrm{d} t+\right.} \\
\left(c_{m m}+C_{R}\right)\left(\int_{0}^{T} \bar{F}_{\text {aew }}(x) \gamma_{e w}(x) \mathrm{d} x-F_{\text {aew }}(T)\right)+ \\
\left.c_{p} \bar{F}_{\text {aew }}(T)+c_{r} F_{\text {aew }}(T)\right] .
\end{gathered}
$$

Remark 1 When $k=0, \theta \rightarrow 0$ and $\Re \rightarrow 0$, (20) can be rewritten as

$$
D(0, T)=\frac{M_{1}+c_{p} \bar{H}(T)+c_{r} H(T)}{\left(\int_{0}^{w} \bar{F}(x) \mathrm{d} x\right) /(\bar{F}(w))+\int_{0}^{T} \bar{H}(x) \mathrm{d} x}
$$

where $\bar{H}(x)=\frac{\bar{F}(w+x)}{\bar{F}(w)}, H(x)=1-\bar{H}(x)$ and $M_{1}=\frac{F(w) c_{0}}{\bar{F}(w)}$.

The extended warranty period and repair-cost threshold both are not considered in this model, so the maintenance policy in this model is a classical age replacement policy after the warranty expiration, which is similar to [9].

Remark 2 When $w=0, k=0, \theta \rightarrow 0$ and $\Re \rightarrow 0$, (20) can be rewritten as

$$
D(0, T)=\frac{c_{p} \bar{F}(T)+c_{r} F(T)}{\int_{0}^{T} \bar{F}(x) \mathrm{d} x} .
$$

Without considering any extended warranty period and repair-cost threshold, maintenance policy in this model is a classical age replacement policy for a new product.

\subsection{Optimization}

In this subsection, we will find the optimal $T^{*}$ and $\Re^{*}$ by minimizing the expected cost rate. This is a single-variable optimization problem, wherein each of both is optimized based on the other variable to be fixed.

We rewrite (20) as

$$
\begin{gathered}
D(\Re, T)=\left[a+\left(c_{2}+C_{R}\right) .\right. \\
\left(\int_{0}^{T} \bar{F}_{\text {aew }}(x) \gamma_{\text {ew }}(x) \mathrm{d} x-F_{\text {aew }}(T)\right)+ \\
\left.c_{p} \bar{F}_{\text {aew }}(T)+c_{r} F_{\text {aew }}(T)\right] \cdot \frac{1}{b+\int_{0}^{T} \bar{F}_{\text {aew }}(x) \mathrm{d} x} \quad \text { (23) } \\
\text { where } a=M+k c_{e}+c_{\text {ew }} \int_{w}^{k \xi} \gamma(t) \mathrm{d} t \text { and } b= \\
\left(\int_{0}^{w} \bar{F}_{\theta}(x) \mathrm{d} x\right) / \bar{F}_{\theta}(w)+k \xi . \\
\text { We can differentiate }(23) \text { with respect to } T \text { and the first- } \\
\text { order derivative of } D(\Re, T) \text { at } T \text { is given by } \\
\frac{\mathrm{d} D(\Re, T)}{\mathrm{d} T}=\frac{1}{b+\int_{0}^{T} \bar{F}_{\text {aew }}(x) \mathrm{d} x}\left[\left(c_{2}+C_{R}\right) .\right. \\
\left.\left(\bar{F}_{\text {aew }}(T) \gamma_{\text {ew }}(T)-f_{\text {aew }}(T)\right)-c_{p} f_{\text {aew }}(T)+c_{r} f_{\text {aew }}(T)\right]-
\end{gathered}
$$




$$
\begin{gathered}
{\left[a+\left(c_{2}+C_{R}\right)\left(\int_{0}^{T} \bar{F}_{\text {aew }}(x) \gamma_{e w}(x) \mathrm{d} x-F_{\text {aew }}(T)\right)+\right.} \\
\left.c_{p} \bar{F}_{\text {aew }}(T)+c_{r} F_{\text {aew }}(T)\right] \cdot \frac{\bar{F}_{\text {aew }}(T)}{\left(b+\int_{0}^{T} \bar{F}_{\text {aew }}(x) \mathrm{d} x\right)^{2}}
\end{gathered}
$$

where $f_{\text {aew }}(T)=\frac{\mathrm{d} F_{a e w}(T)}{\mathrm{d} T}$. Let

$$
\begin{gathered}
J(T)=\left(b+\int_{0}^{T} \bar{F}_{\text {aew }}(x) \mathrm{d} x\right)\left[\left(c_{2}+C_{R}\right) .\right. \\
\left.\left(\bar{F}_{\text {aew }}(T) \gamma_{\text {ew }}(T)-f_{\text {aew }}(T)\right)-c_{p} f_{\text {aew }}(T)+c_{r} f_{\text {aew }}(T)\right]- \\
{\left[a+\left(c_{2}+C_{R}\right)\left(\int_{0}^{T} \bar{F}_{\text {aew }}(x) \gamma_{e w}(x) \mathrm{d} x-F_{\text {aew }}(T)\right)+\right.} \\
\left.c_{p} \bar{F}_{\text {aew }}(T)+c_{r} F_{\text {aew }}(T)\right] \cdot \bar{F}_{\text {aew }}(T) .
\end{gathered}
$$

Supposing that $J(T)$ is continual with respect to $T$ and $J(\infty) \lim _{T \rightarrow \infty} J(T)$ exists. We have

$$
\begin{gathered}
J(0)=\lim _{T \rightarrow 0} J(T)=b\left[\left(c_{2}+C_{R}\right)\left(\gamma_{e w}(0)-f_{\text {aew }}(0)\right)+\right. \\
\left.\left(c_{r}-c_{p}\right) f_{\text {aew }}(0)\right]-\left(a+c_{p}\right) .
\end{gathered}
$$

Theorem 1 For any fixed $\Re \geqslant 0$, the following results hold.

(i) If $J(0) \leqslant 0(J(0) \geqslant 0)$ and $J(\infty) \geqslant 0(J(\infty) \leqslant 0)$, then there exists a finite $T^{*}$ minimizing $D(\Re, T)$;

(ii) The condition of (i) holds and $J(T)$ is strictly monotonous, then there exists a unique finite $T^{*}$ minimizing $D(\Re, T)$;

(iii) If $J(T)$ is non-decreasing (or non-increasing) and $J(0)>0$ or $J(\infty)<0($ or $J(\infty)>0$ or $J(0)<0)$, then " $T^{*}=0$ " or " $T^{*}=\infty$ " is the optimal value of (20).

Theorem 1 gives the existence and uniqueness of the optimal PR age $T^{*}$, which minimizes the expected cost rate. We can also present the existence and uniqueness of the optimal repair-cost threshold $\Re^{*}$ by using the method similar to optimize the PR age $T^{*}$. It is worth pointing out that the existence and uniqueness of the optimal repair-cost threshold $\Re^{*}$ are related to the expression of the distribution function $G(y)$. The different expression leads to a different computation complexity, and derives them if interested.

\section{Numerical example}

To illustrate the model proposed in the previous section, this section presents a simple numerical example. We assume the failure rate function of lifetime is Weibull failure rate function given by

$$
\gamma(x)=\beta \alpha(\beta x)^{\alpha-1} \text { with } \alpha=2 \text { and } \beta=0.5 .
$$

And we assume that the repair cost conforms to the exponential distribution function given by $G(y)=1-$ $\exp (-\lambda t)$ with $\lambda=0.3$.

The following parameter values, $\theta=1, \xi=0.2$, $c_{e}=1, c_{p}=10, c_{r}=15$ and $c_{0}=c_{1}=c_{2}=0.5$ are used in this example. Using the Matlab software, we can obtain the following results.

Table 1 Optimal preventive replacement age $T^{*}$ and repair-cost threshold $\Re^{*}$

\begin{tabular}{ccccc}
\hline$w$ & $k$ & $T^{*}$ & $\Re^{*}$ & $D\left(\Re^{*}, T^{*}\right)$ \\
\hline \multirow{3}{*}{0.8} & 0 & 2.6925 & 3.4334 & 5.9312 \\
& 1 & 2.6179 & 3.4239 & 6.1415 \\
& 2 & 2.5253 & 3.4025 & 6.3176 \\
\hline \multirow{2}{*}{1} & 0 & 2.3448 & 3.3201 & 5.6526 \\
& 1 & 2.2592 & 3.3016 & 5.8418 \\
& 2 & 2.1569 & 3.2743 & 6.0008 \\
\hline \multirow{2}{*}{1.2} & 0 & 1.9500 & 3.1953 & 5.3007 \\
& 1 & 1.8549 & 3.1719 & 5.4736 \\
& 2 & 1.7446 & 3.1424 & 5.6200 \\
\hline
\end{tabular}

From Table 1, we can derive the following remarks.

(i) For the same $k$, the optimal PR age $T^{*}$, the optimal repair-cost threshold $\Re^{*}$ and the expected cost rate $D\left(\Re^{*}, T^{*}\right)$ are decreasing with respect to $w$. The decreasing PR age $T^{*}$ indicates that the replacement actions are more frequent with respect to $w$. The decreasing expected cost rate $D\left(\Re^{*}, T^{*}\right)$ implies that it is more sensible to purchase the product with a longer warranty period.

(ii) For the same $w$, the optimal PR age $T^{*}$ and the optimal repair-cost threshold $\Re^{*}$ decrease with respect to $k$, while the expected cost rate $D\left(\Re^{*}, T^{*}\right)$ is increasing. The decreasing PR age $T^{*}$ means that replacement actions are more frequent with respect to the number of the extended warranty period $k$. When more units of the extended warranty period are bought, the expected cost rate $D\left(\Re^{*}, T^{*}\right)$ is increasing. These monotonous regularities imply that the consumer should purchase few units of the extended warranty period in order to reduce the expected cost rate and replacement frequency.

\section{Conclusions}

In this paper, we consider an RFRW policy with the repaircost threshold. From the consumer's perspective, then, we present an optimal replacement policy after the extended warranty by considering a combination of the age-based PR and cost-based CR. The life cycle is, from the consumer's perspective, defined as the time between two consecutive replacements executed by the consumer and com- 
posed of the basic warranty, extended warranty and PR period. During the PR period, the consumer performs the CR if the repair cost resulted from failure exceeds a repaircost threshold; otherwise, the consumer carries out the PR for the product at the PR age. The expected cost rate model is derived, incorporating costs related to failure, repair and replacement. The existence and uniqueness of the optimal solution that minimizes the expected cost rate are presented. In addition, a numerical example is presented to demonstrate the result obtained in this study. From the numerical example, we can conclude that the consumer should purchase sensibly the product with a longer warranty period and purchase few units of the extended warranty period.

The maintenance policy proposed in this paper can be utilized to guide the consumer how to maintain the reliability of the product after the extended warranty expires. Additionally, this paper provides the theoretical support for the manufacturer to make a choice between the renewable warranty policy with a repair cost limit and the traditional renewable warranty policy.

\section{References}

[1] D. N. P. Murthy, W. R. Blischke. The system warranty management - III: a review of mathematical models. European Journal of Operational Research, 1992, 63(1): 1-34.

[2] D. N. P. Murthy, D. G. Nguyen. An optimal repair cost limit policy for servicing warranty. Mathematical and Computer Modelling, 1988, 11: 595-599.

[3] Y. Huang, W. Gau, J. Ho. Cost analysis of two-dimensional warranty for products with periodic preventive maintenance. Reliability Engineering and System Safety, 2015, 134: 51 - 58.

[4] Y. Wang, Z. Liu, Y. Liu. Optimal preventive maintenance strategy for repairable items under two-dimensional warranty. $R e$ liability Engineering and System Safety, 2015, 142: 326-333.

[5] D. Yang, Z. He, S. He. Warranty claims forecasting based on a general imperfect repair model considering usage rate. Reliability Engineering and System Safety, 2016, 145: 147-154.

[6] K. M. Jung, M. Park, D. H. Park. Cost optimization model following extended renewing two-phase warranty. Computers and Industrial Engineering, 2015, 79: 188 - 194.

[7] K. J. Chung. Optimal repair-cost limit for a consumer following expiry of a warranty. Microelectronics Reliability, 1994, 34(10): $1689-1692$.

[8] J. S. Dagpunar, N. Jack. Optimal repair-cost limit for a consumer following expiry of a warranty. IMA Journal of Management Mathematics, 1992, 4(2): 155-161.

[9] I. Sahin, H. Polatoglu. Maintenance strategies following the expiration of warranty. IEEE Trans. on Reliability, 1996, 45(2): $220-228$.
[10] K. M. Jung, M. J. Park, D. H. Park. System maintenance cost dependent on life cycle under renewing warranty policy. Reliability Engineering and System Safety, 2010, 95(7): 816-821.

[11] G. M. Jung, J. H. Lee, D. H. Park. Periodic preventive maintenance policies following the expiration of warranty. Asia Pacific Journal of Operational Research, 2000, 17(1): 17-26.

[12] G. M. Jung, D. H. Park. Optimal maintenance policies during the post warranty period. Reliability Engineering and System Safety, 2003, 82(2): $173-185$.

[13] M. J. Park, K. M. Jung, D. H. Park. Optimal post - warranty maintenance policy with repair time threshold for minimal repair. Reliability Engineering and System Safety, 2013, 111: $147-153$.

[14] L. Shang, S. Si, Z. Cai. Optimal maintenance-replacement policy of products with competing failures after expiry of the warranty. Computers and Industrial Engineering, 2016, 98: $68-77$.

[15] S. Wu, P. Longhurst. Optimising age-replacement and extended non-renewing warranty policies in lifecycle costing. International Journal of Production Economics, 2011, 130(2): $262-267$.

[16] K. M. Jung, M. J. Park, D. H. Park. System maintenance cost dependent on life cycle under renewing warranty policy. Reliability Engineering and System Safety, 2010, 95(7): 816-821.

[17] R. Barlow, F. Proshan. Mathematical theory of reliability. New York: Wiley, 1965.

[18] S. M. Ross. Applied probability models with optimization application. San Francisco: Holden-Day, 1970.

\section{Biographies}

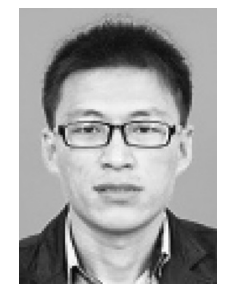

Lijun Shang was born in 1984. He is currently a Ph.D. student in the School of Mechanical Engineering, Northwestern Polytechnical University. He received his M.S. degree from Shijiazhuang Tiedao University in 2014. His research interests include warranty cost analysis, maintenance decision and importance measure of component. E-mail: lijunshang@mail.nwpu.edu.cn

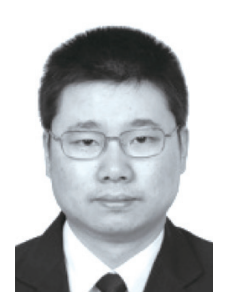

Zhiqiang Cai was born in 1981. He is currently a professor in the School of Mechanical Engineering, Northwestern Polytechnical University, China (NPU). He is also a member of IEEE and IEEE Reliability Society. He received his B.S. and M.S. degrees in 2003 and 2006 at NPU. In 2007, he went to the Ecole Centrale Paris, France as a co-supervised Ph.D. student for one year. In 2010, he received his Ph.D. degree at NPU, majored in management science and engineering. He has published over 20 academic papers in journals and conferences in the past five years. His research topics include maintenance management, failure prediction, Bayesian network modeling and decision making support.

E-mail: caizhiqiang@nwpu.edu.cn 